\title{
Zur Geschichte der konfessionellen Ausnahmeartikel im schweizerischen Verfassungsrecht
}

René Pahud de Mortanges

\section{Einleitung}

Der Bund stellt sich über die religiösen Gemeinschaften und ihre Benennungen. Er anerkennt keine derselben. Er kennt dieselben nur, um ihre Freiheit zu schüzen und um dafür zu sorgen, dass der Friede unter innen herrsche. Er vertheidigt weder eine Konfession noch eine Kirche; er vertheidigt lediglich das Individuum, indem er diesem die Respektirung seines Glaubens und die Freiheit seines Gewissens sichert. ${ }^{1}$

Was hier im Jahr 1873 scheinbar beiläufig in die Botschaft des schweizerischen Bundesrates zur Revision der Bundesverfassung von 1848 eingeflochten wurde, war in Wirklichkeit ein fundamentaler Programmsatz für die Politik des Bundes in religiösen Fragen. Der Bund betrachtete es einzig als seine Aufgabe, den religiösen Frieden zu wahren und die religiöse Freiheit der Religionsgemeinschaften sowie der Bürger zu schützen. Darüber hinaus aber sollte - jedenfalls auf Ebene des Bundesstaates - eine Trennung von Staat und Religionsgemeinschaften bestehen. Es waren vielmehr die Kantone, die das rechtliche Verhältnis zu den Religionsgemeinschaften regelten. Dieses religionsrechtliche Konzept, das zwar bereits zuvor so praktiziert wurde, hier aber erstmals klar formuliert wurde, besteht bis heute. Im geltenden Verfassungsrecht findet es seinen Niederschlag in den Artikeln 15 und 72 der schweizerischen Bundesverfassung.

Die Textpassage, auf dem Höhepunkt des Kulturkampfes in der Schweiz formuliert, ist aber auch deswegen bemerkenswert, weil sie zwischen verschiedenen Erläuterungen zu neuen Verfassungsbestimmungen steht, mit denen sich die Organe des Bundes alles andere als „über die religiösen Gemeinschaften“ stehend erwiesen. Vielmehr wurden eine Reihe von Bestimmungen vorgeschlagen - und mit der Verfassungsrevision 1874 dann auch in Kraft gesetzt - die darauf hinaus liefen, die römisch-katholische Kirche handfest zu diskriminieren. Ihr Einfluss auf die Gesellschaft und den Staat sollte zurück gebunden und eingegrenzt werden.

\footnotetext{
${ }^{1}$ Bundesblatt der Schweizerischen Eidgenossenschaft (BBI) 1873 II 965.
} 
Diese gegen die römisch-katholische Kirche gerichteten

Verfassungsbestimmungen - in der historischen und verfassungsrechtlichen Literatur meist als konfessionelle Ausnahmebestimmungen bezeichnet - sollen im Folgenden näher beleuchtet werden. Die ersten, mehr punktuellen Ausnahmebestimmungen wurden schon bei der Errichtung des Bundesstaates im Jahre 1848 eingeführt, denn auch damals gab es bereits Konflikte zwischen der katholischen Kirche und dem Staat. Doch sollte sich dies erst als Vorgeplänkel erweisen. Anlässlich der Verfassungsrevision von 1874 feuerten die damals liberal und radikal ${ }^{2}$ dominierten Bundesbehörden eine wahre Breitseite von Säkularisierungsbestimmungen gegen die römisch-katholische Kirche ab. Im Gefolge wurden verschiedene Bereiche des öffentlichen Lebens der Normierung durch die römisch-katholische Kirche enthoben und damit ihrer direkten Einflussnahme entzogen.

Auch wenn der Kulturkampf nach 1874 bald wieder abflaute, hat die Kirche die Regelungshoheit in diesen Gebieten nicht mehr zurückerhalten. ${ }^{3}$ Die schweizerischen Katholiken mussten noch lange mit den impliziten Diskriminierungen weiterleben. Erst in der zweiten Hälfte des 20. Jahrhundert wurden sie sukzessive abgeschafft; so zuletzt der sogenannte „Bistumsartikel“, der im Jahr 2001 aus der schweizerischen Bundesverfassung gestrichen wurde.

Die Bundesverfassung ist nach 2001 nicht lange ohne einseitige Beschränkungen der Glaubenspraxis von Religionsgemeinschaften geblieben. Bereits 2009 wurde als Resultat einer Volksabstimmung eine Verfassungsbestimmung eingeführt, wonach der Bau von Minaretten untersagt ist (Art. 72 Abs. 3 BV $^{4}$ ); auch in verschiedenen Kantonen wurden seither Einschränkungen für Muslime diskutiert und teilweise auch schon in Kraft gesetzt. Und bereits 1893 wurde ein gegen die Juden gerichtetes Schächtverbot eingeführt (Art. 25bis $\mathrm{aBV}^{5}$ ), welches 1973 zwar in das Tierschutzgesetz verschoben wurde, der Sache nach aber weiterhin gilt. ${ }^{6}$ So gibt es denn, wie die Forschergruppe um den Berner Politologen Adrian Vatter detailliert analysiert hat, in der schweizerischen Verfassungsgeschichte eine unrühmliche

\footnotetext{
2 Zur Bedeutung dieser Begriffe: Bouquet, Jean-Jacques: Art. Liberalismus, ins Deutsche übersetzt von Alice Holenstein-Beereuter, in: Historisches Lexikon der Schweiz. Bd. 7, Basel 2008, S. 823-827; Tanner, Albert: Art. Radikalismus, in: Historisches Lexikon der Schweiz. Bd. 10, Basel 2011, S. 61-62.

${ }^{3}$ Die Einhaltung ihrer Normen zum Beispiel im Bereich des Eherechtes ist fakultativ, für den Staat nicht mehr relevant.

${ }^{4}$ Bundesverfassung der Schweizerischen Eidgenossenschaft vom 18. April 1999.

${ }^{5}$ Alte Bundesverfassung der Schweizerischen Eidgenossenschaft vom 29. Mai 1874.

${ }^{6}$ Vgl. dazu nur: Krauthammer, Pascal: Das Schächtverbot in der Schweiz 1854-2000. Die

Schächtfrage zwischen Tierschutz, Politik und Fremdenfeindlichkeit. Zürich 2000.
} 
Traditionslinie der Diskriminierung von religiösen Minderheiten. ${ }^{7}$ Solange wie eine religiöse Minderheit als schlecht integriert gilt und als fremd wahrgenommene Wertvorstellungen vertritt, wird sie im politischen System der direkten Demokratie von der stimmberechtigten Bevölkerung minorisiert. ${ }^{8}$ Auch die Katholiken waren im jungen Bundesstaat - und in verschiedenen Kantonen noch bis weit ins 20. Jahrhundert hinein - eine religiöse Minderheit. Es ist kein Zufall, dass die konfessionellen Ausnahmebestimmungen erst 1973 und danach abgeschafft wurden, zu einem Zeitpunkt, in dem die Katholiken in der breiten Bevölkerung ein akzeptierter Teil der Mehrheitsgesellschaft geworden waren. Auch das wird nachfolgend zu thematisieren sein.

Die heute ältere Generation von Katholiken, die die Abstimmung von 1973 noch miterlebt hat, kann sich noch gut an diese Vorgänge und die damit verbundenen Gefühle erinnern. Für Jüngere hingegen, die mit „9/11“ und die endlosen Diskussionen um den Islam in der Schweiz aufgewachsen sind, ist das hingegen graue, abgelebte Vorzeit. Gerade für sie mag die Geschichte des Kommens und Gehens der gegen die katholische Kirche gerichteten konfessionellen Ausnameartikel einigen Lernwert haben, dies in Hinblick auf die Frage, wie selbst ein ansonsten zivilisierter Staat mit religiösen Minderheiten umgeht.

Die Geschichte der Ausnahmeartikel kann nachfolgend nur in aller Kürze skizziert werden und so, dass die allgemeine Geschichte des Kulturkampfes in der Schweiz ${ }^{9}$ und die schweizerische Verfassungsgeschichte des 19. Jahrhunderts ${ }^{10}$ als bekannt vorausgesetzt werden müssen.

\section{Von der Helvetik bis zur ersten Bundesverfassung 1848}

Die 1874 in die schweizerische Verfassung eingeführten konfessionellen Ausnahmeartikel kamen durchaus nicht out of the blue. Zum einen finden sie Entsprechungen in zeitgenössischen Rechtsnormen anderer, ebenfalls involvierter

\footnotetext{
7 Vgl. Vatter, Adrian: Vom Schächt- zum Minarettverbot. Religiöse Minderheiten in der direkten Demokratie. Zürich 2011.

8 Ebd., S. 282.

${ }^{9}$ Zum Einstieg in dieses Thema: vgl. Bischof, Franz Xaver: Art. Kulturkampf, in: Historisches Lexikon der Schweiz. Bd. 7, Basel 2008, S. 484-486. Mit weiterer Literatur und jetzt: Jorio, Marco: Freie Kirche im freien Staat. Der schweizerische Weg vom Staatskirchentum zur Partnerschaft Kirche - Staat, in: Gerhard Schwarz u. a. (Hg.): Religion, Liberalität und Rechtsstaat. Zürich 2015, S. 189-200, hier 195. ${ }^{10}$ Dazu: Kölz, Alfred: Neuere schweizerische Verfassungsgeschichte. Bd. 1, Bern 1992 und Bd. 2, Bern 2004; Kley, Andreas: Verfassungsgeschichte der Neuzeit. Grossbritannien, die USA, Frankreich, Deutschland und die Schweiz. 3. Aufl., Bern 2013, S. 259-518; Pahud de Mortanges, René:

Schweizerische Rechtsgeschichte. Ein Grundriss. Zürich u. a. 2007, S. 153-273.
} 
Staaten, wie zum Beispiel Deutschland. Zum anderen haben sie eine Vorgeschichte, welche recht besehen noch über die Gründung des Bundesstaates zurückreicht. Schon die 1798 der Eidgenossenschaft von Napoleon aufoktroyierte Verfassung der Helvetischen Republik, war in ihren grundrechtlichen und religionsrechtlichen Bestimmungen geprägt vom Misstrauen gegen die katholische Kirche. So lautete Artikel 6 der helvetischen Verfassung:

\begin{abstract}
Die Gewissensfreiheit ist uneingeschränkt; jedoch muss die öffentliche Äusserung von Religionsmeinungen den Gesinnungen der Eintracht und des Friedens untergeordnet sein. Alle Gottesdienste sind erlaubt, insofern sie die öffentliche Ordnung nicht stören und sich keine herrschende Gewalt oder Vorzüge anmassen. Die Polizei hat die Aufsicht darüber und das Recht, sich nach den Grundsätzen und Pflichten zu erkundigen, die darin gelehrt werden. Die Verhältnisse einer Secte mit einer fremden Obrigkeit sollen weder auf die Staatssachen noch auf den Wohlstand und die Aufklärung des Volkes einigen Einfluss haben.
\end{abstract}

Im Geiste des aufgeklärten Staatskirchentums wurde die Kultusfreiheit hier nur sehr eingeschränkt gewährt. ${ }^{11}$ Fast wie heute in der Volksrepublik China konnte die Polizei auch Glaubensinhalte überprüfen und zensurieren und - gleich wie dort heute noch - wurde der Heilige Stuhl als eine fremde, politische und vor allem bedrohliche Macht empfunden. ${ }^{12}$ Denn mit der im Verfassungstext erwähnten „Secte“ war nichts anderes als die katholische Kirche gemeint. Durch die innerreligiös geforderte Loyalität zum Papst schienen die Katholiken noch einer anderen Autorität neben dem Staat gehorsamspflichtig zu sein und das trug aus der Optik der helvetischen Behörden das Risiko innenpolitischer Destabilisierung in sich. Kein Wunder kam die helvetische Verfassung bei den Katholiken im Lande nicht gut an. Sie trafen sich in ihrer Kritik mit den Föderalisten, die den völlig traditionsfremden helvetischen Einheitsstaat nach französischem Muster ablehnten, sowie mit allen, die sich über Inkompetenz, Misswirtschaft, leere Versprechungen und leere Kassen der helvetischen Behörden ärgerten. ${ }^{13}$ Kein Wunder kollabierte dieser Satellitenstaat von Napoleons Gnaden, kaum hatte letzterer im Herbst 1802 seine militärischen Truppen aus der Schweiz abgezogen.

Der nachfolgende Bundesvertrag von 1815, der bis 1848 Grundlage des Verfassungsrechtes der Eidgenossenschaft sein sollte, gewährte überhaupt keine

\footnotetext{
${ }^{11}$ Vgl. Kley, Andreas/Topinke, Esther: Religionsfreiheit zur Zeit der Helvetik, in: Schweizerisches Jahrbuch für Kirchenrecht 5 (2000), S. 73-100; Jorio, Kirche, S. 192 (wie Anm. 9).

12 Vgl. den Artikel: Kahl, Jürgen: Warnung vor dem Gesichtsverlust, in: Neue Zürcher Zeitung, Nr. 154, 7. Juli 2015, S. 7.

13 Zu den Gründen für das Scheitern der Helvetik siehe: Schaaf, Bernhard: Die Entwicklung der wirtschaftlichen Freiheit in der Schweiz während des Französischen Zeitalters (Helvetik und Mediation 1798-1813). Im Vergleich zu Frankreich vor und während der Revolution. Zürich u. a. 2007.
} 
religiösen Freiheitsrechte mehr. Dies war nun wieder eine Frage des kantonalen Verfassungsrechts. Immerhin wurde in Paragraph 12 „der Fortbestand der Klöster und Capitel, und die Sicherheit ihres Eigentums, so weit es von den Kantonsregierungen abhängt, gewährleistet“.

Katholische Klöster galten im 19. Jahrhundert in liberalen und radikalen Kreisen als Orte der religiösen Fanatisierung, als Aussenposten der katholischen Reaktion. Nicht zufällig nahmen die Ereignisse, die zur Gründung des schweizerischen Bundesstaates führten, ihren Ausgang beim Aargauer Klosterstreit 1841. ${ }^{14} \mathrm{Im}$ Kanton Aargau kam es auf dem Lande zu Unruhen, als eine kantonale Verfassungsrevision eine Verteilung der Grossratssitze zulasten der Katholiken brachte. Die Liberalen machten für diese Unruhen die acht im Kantonsgebiet gelegenen Klöster verantwortlich, weshalb diese auf ihr Betreiben hin vom Grossen Rat aufgehoben wurden. Ein Sturm der Entrüstung im In- wie im Ausland war die Folge.

Auch in anderen Kantonen kam es zu religiös-politischen Spannungen. Im Kanton Luzern berief die konservative Regierung 1844 die Jesuiten, die damals bereits im Schulwesen der Kantone Freiburg, Schwyz und Wallis wirkten. Sie sollten in Luzern das Priesterseminar und die theologische Lehranstalt leiten, sowie die Seelsorge ausüben. Auf diese Weise sollte der Einfluss der Kirche auf Staat und Gesellschaft verstärkt werden.

Nach zwei misslungenen Freischarenzügen bewaffneter Freisinniger Richtung Luzern - welche das Ziel verfolgten, die konservative Luzerner Regierung zu stürzen und die Jesuiten zu vertreiben - schlossen die sieben katholischen Kantone Uri, Schwyz, Unterwalden, Luzern, Zug, Wallis und Freiburg Ende 1845 einen Sonderbund als Defensivallianz gegen befürchtete weitere Angriffe. Da aber ein solches Sonderbündnis gegen den Bundesvertrag von 1815 verstiess, ${ }^{15}$ wurde an der Tagsatzung dessen Auflösung beschlossen. Das Ergebnis war der Sonderbundkrieg, ein wenige Wochen dauernder Feldzug der Tagsatzungsarmee durch mehrere Kantone und eine kurze, aber blutige Entscheidungsschlacht vor den Toren der Stadt Luzern, die die katholischen Truppen schmählich verloren. Die Gunst der Stunde nutzend, zimmerten die liberalen und radikalen Kräfte in den

\footnotetext{
${ }^{14}$ Für das Folgende: Pahud de Mortanges, Rechtsgeschichte, S. 173-179 (wie Anm. 10).

$15 \S 6$ des Bundesvertrages von 1815 lautete: „Es sollen unter den einzelnen Kantonen keine dem allgemeinen Bund oder den Rechten anderer Kantone nachtheilige Verbindungen geschlossen werden."
} 
Wochen und Monaten nach ihrem Sieg in aller Eile einen Entwurf für eine Bundesverfassung. Dieser wurde zwar von einer Mehrheit, aber durchaus nicht von allen Kantonen im Laufe des Jahres 1848 angenommen. Jenen Kantonen, in denen sich die Bevölkerungsmehrheit dagegen aussprach, wurde die Verfassung faktisch aufgezwungen.

Die neue Verfassung enthielt nun ein explizites Jesuitenverbot. Artikel 58 lautete: „Der Orden der Jesuiten und die ihm affilierten Gesellschaften dürfen in keinem Theile der Schweiz Aufnahme finden." Diese Bestimmung, in den Beratungen auf Antrag des Standes Zürich aufgenommen, ${ }^{16}$ war eigentlich überflüssig, zumal die Tagsatzung diese Ordensgemeinschaft bereits im September 1847 aus der Schweiz ausgewiesen hatte. ${ }^{17}$ Geschützt wurde in Artikel 44 immerhin die freie Ausübung des Gottesdienstes „im ganzen Umfange der Eidgenossenschaft“, aber nur für die „anerkannten christlichen Konfessionen“. Keine Gottesdienstfreiheit bestand damit für Juden, aber auch für die Angehörigen von kleineren christlichen Gemeinschaften neben der evangelischen und katholischen Kirche. Namentlich in der Westschweiz existierten grosse Vorbehalte gegen christliche „Sekten“. 18 Überhaupt war man von einer umfassenden Religionsfreiheit noch weit entfernt.

Auch die Niederlassungsfreiheit wurde gemäss Artikel 41 nur „Schweizern, welche einer christlichen Konfession angehören“ gewährt. Das richtete sich primär gegen die Juden, die sich in dieser Zeit nur in den aargauischen Dörfern Endigen und Lengnau niederlassen durften. Indem man ihre Niederlassung auf diese beiden „Judendörfer“ beschränkte, hielt man sich in den grossen Städten wirtschaftliche Konkurrenz fern. Damit aber hatte sich die Schweiz spätestens 1864 in einen unhaltbaren Zustand manövriert. Der in diesem Jahr mit Frankreich geschlossene Handelsvertrag gewährte allen Franzosen die freie Niederlassung und Gewerbeausübung. Die schweizerischen Juden waren damit unvermittelt schlechter gestellt als ihre französischen Glaubensgenossen. In einer ersten, kleinen Teilrevision der Bundesverfassung wurde 1866 die religiöse Einschränkung der Niederlassungsfreiheit gestrichen. ${ }^{19}$ Das ermöglichte in der Folge die Gründung von

\footnotetext{
${ }^{16}$ Kölz, Verfassungsgeschichte, Bd. 1, S. 587 (wie Anm. 10).

17 Bischof, Franz Xaver: Art. Jesuiten, in: Historisches Lexikon der Schweiz. Bd. 6, Basel 2007,

S. 790-792, hier 792 .

${ }^{18}$ Kölz, Verfassungsgeschichte, Bd. 1, S. 586 (wie Anm. 10).

19 Vgl. Pahud de Mortanges, Rechtsgeschichte, S. 179-180 (wie Anm. 10).
} 
jüdischen Gemeinschaften in verschiedenen grösseren und kleineren Städten der Schweiz. ${ }^{20}$

Was die Bundesverfassung von 1848 betrifft, so enthielt diese noch eine weitere, vierte Ausnahmebestimmung. Wahlfähig in den Nationalrat (Art. 64 aBV), aber auch in den Bundesrat (Art. 84 aBV) und das Bundesgericht (Art. 97 aBV) waren nur „stimmberechtigte Schweizerbürger weltlichen Standes“. Das schloss dem Wortlaut nach Geistliche aller Kirchen und anderen Religionsgemeinschaften aus, zielte aber faktisch auf katholische Kleriker. Anders als reformierte Pfarrer konnten diese angesichts der sakramentalen Natur der Priesterweihe nicht einfach für die Dauer des politischen Mandates auf ihr geistliches Amt verzichten. ${ }^{21}$ Die obersten Organe des Bundes sollten mit diesen Inkompatibilitätsbestimmungen frei von katholischem Einfluss bleiben.

\section{Das Exklusions- und Säkularisierungsprogramm der Bundesverfassung} von 1874

1864 verurteilte Papst Pius IX. in der seiner Enzyklika Quanta cura angehängten Liste Syllabus errorum 80 „Irrtümer“ der zeitgenössischen Politik, Wissenschaft und Gesellschaft. Darunter waren auch die individuelle Glaubensfreiheit, der Protestantismus, die Trennung von Kirche und Staat, die Ziviltrauung und die Ehescheidung. 1870 verabschiedete das erste Vatikanische Konzil das Unfehlbarkeitsdogma: Der Papst konnte fortan in bestimmten Konstellationen kirchliche Lehrmeinungen als unfehlbar erklären. Dieses Anti-Programm gegen alle Errungenschaften der Aufklärung, der französischen Revolution und des modernen Lebens führte zu einem Sturm der Entrüstung bei Liberalen, Protestanten aber auch aufgeklärten Katholiken. In den liberal regierten Kantonen kam es zur Spaltung der katholischen Kirche und zur Gründung unabhängiger Kirchgemeinden, welche sich 1876 zur Christkatholischen Kirche zusammenschlossen. ${ }^{22}$ Liberal dominierte Kantonsregierungen und römisch-katholische Bischöfe lieferten sich heftige Auseinandersetzungen.

\footnotetext{
${ }^{20}$ Vgl. Weingarten, Ralph: Gleichberechtigt in die neue Zeit. Die „Gründerzeit" des Schweizer Judentums 1866-1920, in: Willy Guggenheim (Hg.): Juden in der Schweiz. Glaube - Geschichte Gegenwart. Zürich 1982, S. 54-68.

${ }^{21}$ Kraus, Dieter: Schweizerisches Staatskirchenrecht. Tübingen 1993, S. 141.

${ }^{22}$ Vgl. von Arx, Urs: Art. Christkatholische Kirche, in: Historisches Lexikon der Schweiz. Bd. 3, Basel 2004, S. 373-374.
} 
Diese kirchenpolitischen Streitigkeiten fielen genau in die Zeit, in der auf Bundesebene Bestrebungen zur Revision der Bundesverfassung im Gang waren. War eine erste Revision 1866 - abgesehen von der bereits erwähnten Liberalisierung der Niederlassungsfreiheit - vom Stimmvolk abgelehnt worden, verstärkte die Kulturkampfstimmung nun die Dynamik des Revisionsprozesses. ${ }^{23}$ Geschickt wussten die Radikalen das - aus ihrer Optik - provokative Handeln Roms und der schweizerischen Bischöfe für ihre eigenen Ziele zu nutzen.

So verwahrten sich die schweizerischen Bischöfe - verständlich, aber taktisch wohl nicht sonderlich klug - in dramatischen Worten in einer Denkschrift aus dem Jahr 1871 gegen diverse staatliche Einflussnahmen im Bistum Basel, gegen die Zivilehe, die konfessionell gemischten Schulen, gegen die Entheiligung der Sonnund Feiertage, gegen die Aufhebung der Klöster in verschiedenen Kantonen und gegen das Jesuitenverbot. Ihr Fazit war: „Der Geist des Antichristentums schreitet rastlos unter den Völkern einher und bewegt mit seinen Flügelschlägen die Atmosphäre der öffentlichen Meinung zu den frivolsten Verleugnungen aller ewigen Wahrheiten des Christentums." 24

Die Denkschrift war eine Reaktion auf die staatskirchenrechtlichen Bestimmungen in der vorausgegangenen Botschaft des Bundesrates zur Revision der Bundesverfassung von $1870 .{ }^{25}$ Von der liberal und radikal zusammengesetzten Mehrheit im Bundesparlament wurde die bischöfliche Jeremiade aber als Provokation empfunden. ${ }^{26}$ Diese fühlte sich in ihren anti-römischen Affekten bestätigt und nun erst recht legitimiert, den Schutzbereich der Religionsfreiheit auszudehnen und die Zahl der konfessionellen Ausnahmebestimmungen zu Ungunsten der römisch-katholischen Kirche zu vergrössern. ${ }^{27}$ Mit dem gleichzeitig in anderen Bereichen angestrebten Ausbau der Bundeskompetenzen war jedoch das politische Fuder überladen. Nicht nur in den katholisch-konservativen ehemaligen Sonderbundkantonen wurde dieser Verfassungsentwurf in der Volksabstimmung 1872 abgelehnt.

\footnotetext{
${ }^{23}$ Kölz, Verfassungsgeschichte, Bd. 2, S. 519-520 (wie Anm. 10).

${ }^{24}$ Greith, Carl Johann: Die Lage der katholischen Kirche und das öffentliche Recht in der Schweiz. Denkschrift der schweizerischen Bischöfe an die hohe Bundesversammlung bei Anlass der Revision der schweizerischen Bundesverfassung. St. Gallen 1871, S. 91.

25 BBI 1870 II 665-710.

${ }^{26}$ Kölz, Verfassungsgeschichte, Bd. 2, S. 566 (wie Anm. 10).

27 Im Einzelnen: Ebd., S. 567-573.
} 
Der dritte Entwurf, mit dessen Ausarbeitung nach der Ablehnung der zweiten Vorlage sofort begonnen wurde, nahm in Sachen Zentralisierung Verschiedenes wieder zurück, um so das Vertrauen der föderalistischen Kreise in der Westschweiz zu gewinnen. ${ }^{28}$ Den katholisch-konservativen Teilen der Bevölkerung kam man hingegen nicht in gleicher Weise entgegen. ${ }^{29}$

Das staatskirchenpolitische Programm von 1872 blieb hierbei erhalten und wurde sogar noch erweitert:

- Das Jesuitenverbot wurde insofern verstärkt, als den Ordensangehörigen „jede Wirksamkeit in Kirche und Schule untersagt" und der Bund ermächtigt wurde, dieses Verbot auf andere Orden auszudehnen (Art. $51 \mathrm{aBV}$ ).

- Die Errichtung neuer und die Wiederherstellung aufgehobener Klöster und religiöser Orden sollte unzulässig sein (Art. 52 aBV).

- Die geistliche Gerichtsbarkeit sollte abgeschafft sein (Art. 58 Abs. 2 aBV).

- Das Recht zur Ehe wurde unter den Schutz des Bundes gestellt. Dieses Recht sollte weder aus kirchlichen noch aus ökonomischen Rücksichten beschränkt werden dürfen (Art. 54 Abs. 1 und 2 aBV).

- Die Feststellung und Beurkundung des bürgerlichen Standes sollte Sache der weltlichen Behörden sein (Art. 53 Abs. 1).

- Auch die Verfügung über die Begräbnisplätze sollte den bürgerlichen Behörden zustehen und diese hatten dafür zu sorgen, dass jeder Verstorbene „schicklich“ beerdigt wurde (Art. 53 Abs. 2 aBV).

- Die Errichtung von Bistümern wurde der Genehmigung des Bundes unterworfen (Art. 50 Abs. 4 aBV).

- Die Kantone wurden beauftragt, für genügenden Primarschulunterricht zu sorgen, der ausschliesslich unter staatlicher Leitung zu stehen habe. Die öffentlichen Schulen sollten von den Angehörigen aller Bekenntnisse ohne Beeinträchtigung ihrer Glaubens- und Gewissensfreiheit besucht werden können (Art. 27 Abs. 2 und 3 aBV).

Breiten Raum nahm die Gewährung der Religionsfreiheit ein:

- Die Gewissensfreiheit der Bundesverfassung von 1848 wurde nun zu einer umfassende Glaubens- und Gewissensfreiheit erweitert (Art. 49 aBV), welche nicht mehr einzelnen Religionsgemeinschaften vorbehalten sein sollte.

\footnotetext{
${ }^{28}$ Kley, Verfassungsgeschichte, S. 306 (wie Anm. 10).

${ }^{29}$ Kraus, Staatskirchenrecht, S. 37 (wie Anm. 21).
} 
Niemand sollte zur Teilnahme an einer Religionsgemeinschaft, an einem religiösen Unterricht oder zur Vornahme einer religiösen Handlung gezwungen werden können. Wegen Glaubensansichten sollte niemand mit (staatlichen) Strafen belegt werden können. Die Glaubensansichten sollten auch nicht von der Erfüllung der bürgerlichen Pflichten entbinden.

- Separat erwähnt wurde (in Art. 50 Abs. 1 aBV) die Kultusfreiheit; diese sollte innerhalb der Schranken der Sittlichkeit und der öffentlichen Ordnung gewährleistet sein.

Am 19. April 1874 nahmen Volk und Stände die um diese und andere Bestimmungen revidierte Verfassung an. Für die obersten Behörden des Bundes stellten sie auch ein Arbeitsprogramm dar. Dies namentlich in den Bereichen Ehe, Zivilstand und Bestattungswesen. Das Bedürfnis nach Säkularisierung in diesen drei Bereichen bestand nicht zuletzt als Folge der Gewährung der Niederlassungsfreiheit. Diese hatte zu einer sukzessiven konfessionellen Durchmischung der Bevölkerung in den zuvor meistenorts konfessionell klar abgegrenzten Territorien geführt.

Eheschliessungen über die Konfessionsgrenzen hinaus („Mischehen“), wie sie jetzt vermehrt vorkamen, wurden jedoch namentlich von der katholischen Kirche aus Angst vor dem Glaubensverlust der eigenen Mitglieder bekämpft. Diskriminierende Praktiken vor Ort waren daher nicht selten. Um diese zu verhindern, entzog der Staat der Kirche die Kompetenz im Eheschliessungsrecht. Das war ein Regelungsbereich, der jedenfalls in katholischen Gebieten seit Jahrhunderten in kirchlichen Händen gewesen war. ${ }^{30}$ Das 1875 angenommene „Bundesgesetz betreffend Feststellung und Beurkundung des Zivilstandes und der Ehe" führte schweizweit die obligatorische Zivilehe ein, enthielt aber auch schon einige wichtige Regeln des materiellen Eherechts. ${ }^{31}$ In Vollziehungsverordnungen hatten die Kantone nun das neue Recht einzuführen, was nicht selten Schwierigkeiten bereite. Wenn Verstösse gegen die neuen Bestimmungen gerügt wurden, hatten der Bundesrat und das Bundesgericht über die Beschwerden zu entscheiden. Mit dem schweizerischen

\footnotetext{
${ }^{30}$ In reformierten Kantonen gab es mancherorts bereits vor 1875 die obligatorische Zivilehe, wohingegen andere eine bloss fakultative Eheschliessung vor der weltlichen Behörde kannten und sich in verschiedenen katholischen Kantonen die kirchliche Eheschliessung hielt. Vgl. Siffert, Rino: Verlobung und Trauung. Die geschichtliche Entwicklung des schweizerischen Eheschliessungsrechts. Zürich u. a. 2004, S. 95-128.

${ }^{31}$ Dazu: Ebd., S. 138-152; Coing, Helmut: Handbuch der Quellen und Literatur der neueren europäischen Privatrechtsgeschichte. Bd. III/2, München 1982, S. 2039.
} 
Zivilgesetzbuch von 1912 wurde der Rest des materiellen Eherechts landesweit vereinheitlicht.

Schon in den Beratungen zur (abgelehnten) Revisionsvorlage von 1872 war das Recht auf freie Niederlassung und auf freie Ehe mit dem Begräbniswesen verknüpft worden. Wenn man das Recht habe, in der ganzen Schweiz eine Ehe zu schliessen, - so ein Mitglied des Nationalrates - gebiete es die Logik, dass man auch das Recht habe, seine Verwandten dort zu bestatten, wo man niedergelassen sei. ${ }^{32}$ Es könne nicht sein, dass die eine Materie durch den Staat und die andere durch die Kirche geregelt sei. Das zielte auf das meist noch in kirchlicher Hand befindliche Friedhofswesen ab. Auch hier waren es vorwiegend diskriminierende Praktiken auf katholischen Friedhöfen, die Anlass zur Sorge gaben: Andersgläubige, Ausgetretene und Selbstmörder wurden dort manchmal gar nicht oder in einer abgegrenzten Abteilung oder dann unter Verweigerung der ortsüblichen Feierlichkeiten bestattet. Das sollte nicht mehr vorkommen; jeder Verstorbene sollte von nun an „schicklich“ bestattet werden. Wie die Rekurspraxis des Bundesrates in der Folgezeit zeigt, ging es ihm dabei weniger um die Verhinderung einvernehmlicher konfessioneller Separierung als um die Verhinderung von Diskriminierung. Gleichwohl wurden nun in manchen Gemeinden neben den konfessionellen Friedhöfen kommunale Friedhöfe errichtet, auf denen die Verstorbenen ungeachtet ihrer Konfession der Reihe nach bestattet wurden.

\section{Der lange Weg zurück}

Verweltlichtes Schul-, Ehe-, Zivilstands- und Bestattungswesen - die Säkularisierung dieser Bereiche ist seither nicht rückgängig gemacht worden. Auch die letzten Reste der in einigen katholischen Gebieten noch praktizierten geistlichen Gerichtsbarkeit in Ehesachen wurden nach 1874 von der Bundesgerichtspraxis sukzessive beseitigt. Die Schweiz ist bezüglich der Säkularisierung des Rechts unter dem Einfluss des Kulturkampfes weiter gegangen als andere europäische Länder, wo die traditionellen Kompetenzen der Kirchen länger erhalten blieben. Von Bedeutung ist hier wohl die Nähe zu Frankreich gewesen, wo diese Rechtsbereiche bereits mit dem „Code civil“ von 1804 in staatliche Hand übergegangen waren und somit ein Modell bestand, das man übernehmen konnte.

\footnotetext{
32 Vgl. im Einzelnen: Pahud de Mortanges, René: Die historische Entwicklung des staatlichen Bestattungsrechts in der Schweiz (im Druck).
} 
Die Vereinheitlichung des Rechts in einem so zentralen Lebensbereich wie den der Ehe hat vermutlich für die Folgezeit eine nicht geringe integrative Rolle gespielt: Katholiken und Protestanten lebten nun nach demselben Recht und damit nach denselben, diesem Recht zugrundliegenden (christlichen) Werten und Anschauungen.

Anders war es mit den einseitig auf die katholische Kirche zielenden Ausnahmebestimmungen. Sie waren fortan ein Stachel im Fleisch der Katholiken und verzögerten die Überwindung konfessioneller Trennungen. In den ersten Jahrzehnten nach Inkrafttreten der Bestimmungen und noch zu Beginn des 20. Jahrhunderts waren die Behörden um die strikte Einhaltung der Verbote bemüht. Jesuiten beispielsweise durften auch nicht bloss einmal und vertretungsweise in einer Kirche predigen; ebenso wurde ihnen untersagt, Vorträge zu halten, dies selbst im privaten Kreis.

Staatsrechtler in der ersten Hälfte des 20. Jahrhunderts kommentierten etwa das Kloster- und Jesuitenverbot je nach eigener konfessioneller Provenienz diametral entgegensetzt.

Ulrich Lampert (1865-1947), zum Katholizismus konvertierter Professor unter anderem für Kirchenrecht an der Universität Freiburg i. Ue. ${ }^{33}$ empörte sich 1938 in seinem monumentalen Werk „Kirche und Staat in der Schweiz“:

Der Jesuitenorden ist die Zielscheibe eines unbegründeten und unvernünftigen Hasses; er ist fortgesetzten allseitigen heftigsten Verfolgungen seit dem 16. Jahrhundert ausgesetzt, trotzdem die gegen ihn vorgebrachten Vorurteile und Anfechtungen längst öfters widerlegt worden sind. ${ }^{34}$ [...] Die grossen allgemeinen Leistungen des vielgeschmähten und vielgehassten Ordens können in seinen gegnerischen Kreisen nicht auf unbefangene Würdigung rechnen bis nicht das ungeheuerliche Zerrbild verschwindet, das die Gegnerschaft vom Orden seit alter Zeit sich zurechtgelegt hat. ${ }^{35}$

Anders beurteilen die Situation hingegen Fritz Fleiner (1867-1937) und Zaccaria Giaccometti (1893-1970), Professoren an der Universität Zürich, die diese Bestimmungen als notwendig erachten, um den religiösen Frieden zu wahren. ${ }^{36}$

Nach dem Zweiten Weltkrieg verloren die konfessionellen Gegensätze zunehmend an Bedeutung. Im Zuge der Verfolgung durch den Nationalsozialismus in

\footnotetext{
33 Über ihn: Carlen, Louis: Kirchenrecht und Kirchenrechtslehrer an der Universität Freiburg i. Ue. Freiburg i. Ue. 1979, S. 43-48.

${ }^{34}$ Lampert, Ulrich: Kirche und Staat in der Schweiz. Bd. 2, Freiburg i. Ue. 1938, S. 428.

35 Ebd., S. 429.

36 Jorio, Marco: Art. Ausnahmeartikel, in: Historisches Lexikon der Schweiz. Bd. 1, Basel 2002, S. 590; Kley, Andreas: Von Stampa nach Zürich. Der Staatsrechtler Zaccaria Giacometti, sein Leben und Werk und seine Bergeller Künstlerfamilie. Zürich 2014, S. 326.
} 
Deutschland hatten Jesuiten in der Schweiz Asyl gefunden. Die Haltung der Behörden ihnen gegenüber wurde toleranter. Namentlich ihre akademische und seelsorgerliche Tätigkeit an Hochschulen wurde nun geduldet. Auch die Verlegung und Vergrösserung von Klostergemeinschaften wurde fortan toleriert. ${ }^{37}$

Das Zweite Vatikanische Konzil (1963-1965) präsentierte die katholische Kirche als eine auf Modernisierung bedachte Institution. In der Erklärung über die Religionsfreiheit von 1965 anerkannte die Kirche ausdrücklich die (Selbst)Verpflichtung des Staates, die Glaubens- und Gewissensfreiheit zu schützen. Das Konzilsdekret über den Ökumenismus von 1964 war getragen vom Willen zur Toleranz und zur Zusammenarbeit zwischen den christlichen Konfessionen. In derselben Zeit bemühte sich die Schweiz ihrerseits um eine Ratifizierung der Europäischen Menschenrechtskonvention (EMRK) von 1950. Kloster- und Jesuitenartikel bildeten dafür jedoch ein Hindernis zumal die einseitige Einschränkung der Freiheit einer Religionsgemeinschaft gegen verschiedene Regelungen der EMRK verstiess. Gestützt auf das Gutachten ${ }^{38}$ des angesehen Zürcher Rechtsprofessors Werner Kägi (1909-2005) ${ }^{39}$ beantragte der Bundesrat in seiner Botschaft von $1971^{40}$ die ersatzlose Aufhebung dieser Vorschriften. ${ }^{41}$ Neben Kägi unterstützten auch andere prominente Reformierte die Streichung. Der Abstimmungskampf im Jahr 1973 währte nur wenige Monate, wurde aber emotional und zum Teil mit konfessionalistischen Argumenten geführt. In der darauffolgenden Volksabstimmung wurde die Streichung mit 55 zu 45 Prozent gutgeheissen. Im selben Jahr wurde die EMRK von der Schweiz ratifiziert.

Seit den 1980er Jahren gab es Bestrebungen, die Bundesverfassung einer Totalrevision zu unterwerfen, dies unter anderem um den durch die vielen Einzelrevisionen unübersichtlich gewordenen Verfassungstext formal zu bereinigen. Der 1995 vom Bundesrat vorgeschlagene Entwurf sah eine radikal entschlankte Formulierung der Glaubens- und Gewissenfreiheit vor (der heutige Art. 15 BV).

\footnotetext{
37 Vgl. die geschilderte Praxis in der Botschaft des Bundesrates an die Bundesversammlung über die Aufhebung des Jesuiten- und des Klosterartikels vom 23. Dezember 1971, in: BBI 1972 I 138-141.

38 Kägi, Werner: Gutachten zum Jesuiten- und Klosterartikel der Bundesverfassung. Bern 1973.

${ }^{39}$ Vgl. Schmid, Bruno: Art. Kägi. Werner (Ziff. 9), in: Historisches Lexikon der Schweiz. Bd. 7, 2008, S. 37 .

40 Das Gutachten wird in der Botschaft in seinen wichtigsten Argumenten referiert. Vgl. BBI 1972 I 144-157.

${ }^{41}$ Die Arbeit an der Vorlage war initiiert worden durch die 1954 eingereichte Motion des damaligen Obwaldner Ständerates und nachmaligen Bundesrates Ludwig von Moos (1910-1990). Vgl. Feusi Widmer, Roswitha: Art. Moos. Ludwig von (Ziff. 12), in: Historisches Lexikon der Schweiz. Bd. 8, 2009, S. 706. Von Moos studierte von 1930 bis 1933 Rechtswissenschaft an der Universität Freiburg i. Ue. und wird hier auch zu den Studenten von Ulrich Lampert gehört haben.
} 
Entgegen dem ausgewiesenen „rein formalen“ Revisionszweck wurden hier und dort auch materielle Änderungen vorgenommen. So fanden sich im neuen Text weder die alten Inkompatibilitätsbestimmungen für die Wahl in die obersten Bundesbehörden noch das Gebot der schicklichen Bestattung. Während die ersteren stillschweigend als überholt und obsolet beurteilt wurden, betrachtete man das Gebot der schicklichen Bestattung nun im allgemeinen Prinzip der Menschenwürde (Art. 7 BV) mitenthalten. ${ }^{42}$

Indes wagte der Bundesrat nicht, die letzte verbleibende Ausnamebestimmung, den „Bistumsartikel“ (Art. 72 Abs. 3 BV) ${ }^{43}$ zur Streichung vorzuschlagen. Das langjährige, staatspolitisch nicht einfach zu realisierende Projekt der Verfassungsrevision sollte nicht mit einem konfessionell heiklen Traktandum belastet werden. Allerdings liessen die Bundesbehörden nichts anbrennen: Kaum war die Verfassung angenommen und in Kraft getreten, wurde im Parlament eine Motion zur Streichung eingereicht, welche vom Bundesrat unterstützt und in einer Volksabstimmung im Jahr 2001 angenommen wurde. Die geringe Stimmbeteiligung zeigt, dass das Thema nur mehr Wenige interessierte. ${ }^{44}$

Der damit obsolet gewordene Art. 72 Abs. 3 BV sollte freilich nicht lange unbelegt bleiben. Kaum war diese Abstimmung erfolgt, begann eine ganz neue Diskussion, nämlich jene um die Rolle des Islam in der Schweizer Gesellschaft. Diese führte acht Jahre später, im Jahr 2009, zur Abstimmung über das Minarettbauverbot. Dieselbe Diskussion hält bis heute an, wobei sie sich oft an den nach Aussen sichtbaren Symbolen wie zum Beispiel dem Kopftuch abarbeitet: Wenn man schon die Glaubenspraxis der Muslime nicht verbieten kann, so will man sie wenigstens nicht sehen. Dabei sollte uns die Geschichte der konfessionellen Ausnahmeartikel doch lernen, dass einseitige Einschränkungen der Religionsfreiheit meist nichts nützen, aber durchaus schaden können.

\footnotetext{
42 Pahud de Mortanges, Entwicklung (wie Anm. 32).

43 Zu dieser Bestimmung: Winzeler, Christoph: Strukturen von einer „anderen Welt“.

Bistumsverhältnisse im schweizerischen Bundesstaat 1848-1998, ihr historischer Wandel und ihre Inkulturation. Freiburg i. Ue. 1998.

44 Den Schreibenden streifte im Vorfeld der Abstimmung noch der Luftzug ehemaligen konfessionellen Haders. Ein von ihm mitverfasstes Gutachten verneinte die Frage, ob der Bistumsartikel a priori völkerrechtswidrig sein, so wie es dies der Bundesrat behauptete. Das brachte inm erregte Beschimpfungen aufgebrachter Katholiken ein.
} 